\title{
Quantum dots in imaging, drug delivery and sensor applications
}

\author{
This article was published in the following Dove Press journal: \\ International Journal of Nanomedicine \\ 28 July 2017 \\ Number of times this article has been viewed
}

\author{
Cristian T Matea ${ }^{\mathrm{l}, *}$ \\ Teodora Mocan ${ }^{1,2, *}$ \\ Flaviu Tabaran 1,3,* \\ Teodora Pop ${ }^{1,4, *}$ \\ Ofelia Mosteanul,4,* \\ Cosmin Puia ${ }^{1,5, *}$ \\ Cornel lancu ${ }^{1,5, *}$ \\ Lucian Mocan ${ }^{1,5, *}$
}

'Nanomedicine Department, Regional Institute of Gastroenterology and Hepatology "Octavian Fodor", ${ }^{2}$ Department of Physiology, University of Medicine and Pharmacy, "luliu Hatieganu", ' ${ }^{3}$ Department of Pathology, Faculty of Veterinary Medicine, University of Agricultural Sciences and Veterinary Medicine, ${ }^{4}$ Department of Gastroenterology, ${ }^{5}$ Department of Surgery, University of Medicine and Pharmacy, "luliu Hatieganu", Cluj-Napoca, Romania

*These authors contributed equally to this work
Correspondence: Teodora Mocan Department of Physiology, University of Medicine and Pharmacy, "Iuliu Hatieganu", Victor Babes Street 8, ClujNapoca, 4000 I2, Romania

Tel +40 264439696

Email teodora.mocan@umfcluj.ro

\begin{abstract}
Quantum dots (QDs), also known as nanoscale semiconductor crystals, are nanoparticles with unique optical and electronic properties such as bright and intensive fluorescence. Since most conventional organic label dyes do not offer the near-infrared $(>650 \mathrm{~nm})$ emission possibility, QDs, with their tunable optical properties, have gained a lot of interest. They possess characteristics such as good chemical and photo-stability, high quantum yield and size-tunable light emission. Different types of QDs can be excited with the same light wavelength, and their narrow emission bands can be detected simultaneously for multiple assays. There is an increasing interest in the development of nano-theranostics platforms for simultaneous sensing, imaging and therapy. QDs have great potential for such applications, with notable results already published in the fields of sensors, drug delivery and biomedical imaging. This review summarizes the latest developments available in literature regarding the use of QDs for medical applications.
\end{abstract}

Keywords: quantum dots, biomedical applications, nanoprobes, theranostic platforms

\section{Introduction}

Quantum dots (QDs), also known as nanoscale semiconductor crystals, were first described by Ekimov and Onushenko ${ }^{1}$ in a glass matrix, back in 1981, with the first biological imaging application reported in $1998 .{ }^{2}$ Since then the field of QDs has been growing steadily and now includes applications in fields of solar cells, photovoltaic devices, light-emitting diode (LED) fabrication, photodetectors, computing, biomedical imaging and so on.

Most conventional organic label dyes do not offer the near-infrared (NIR) $(>650 \mathrm{~nm})$ emission possibility; this region is highly desired for biomedical imaging due to its reduced light scattering and low tissue absorption, it is why QDs with their tunable optical properties have gained a lot of interest. ${ }^{3}$ These nanometric semiconductors offer unique and fascinating optical properties, such as high quantum yield, size-tunable light emission and good chemical and photo-stability. ${ }^{4}$ QDs, with sizes $2-10 \mathrm{~nm}$, are generally composed of elements (such as $\mathrm{Cd}, \mathrm{Pb}, \mathrm{Hg}$ ) from groups II-VI, III-IV and IV-VI in the periodic table. More recently, researchers have developed ternary I-III-VI QDs (where I = Cu or Ag, III = Ga or In, VI = S or Se). ${ }^{5}$ Different types of QDs can be excited with the same light wavelength, and their narrow emission bands can be detected simultaneously for multiple assays. The fluorescence bands of QDs are dependent on their composition, size and shell thickness. ${ }^{6}$ If the particle size is smaller than the bulk materials' Bohr radius, it causes the energy levels to pose atom-like properties and become discrete compared to the continuum energy levels observed in bulk materials; in other words, these unique properties result from the confinement of 
the states of charge carriers by the physical reduction in the size of the nanoparticles. ${ }^{7}$ In order to use QDs under ambient conditions, they must be stabilized or passivated, because of their high reactivity and surface area. When choosing a stabilizer, one has to take into account the absorption and luminescence spectra profile; also the quantum yield and the lifetime depend on the environment composition. ${ }^{8}$

There is an increasing interest in the development of nano-theranostics platforms for simultaneous sensing, imaging and therapy. QDs have great potential for such applications, with promising results published in the fields of sensors, drug delivery and biomedical imaging. The present paper summarizes the latest developments available in literature regarding the use of QDs for the aforementioned medical applications.

\section{Imaging}

Although fluorescence imaging has been used in animal models, this approach is limited by the poor transmission of visible light through the biological tissue. This has prompted researchers to make use of the NIR optical window (700-1,700 nm) in order to conduct deep-tissue optical imaging with the aid of QDs. ${ }^{9,10}$ A large number of formulations for QDs bio-imaging is currently available in literature.

Kim et al ${ }^{11}$ proposed polydentate phosphine-coated QDs for major cancer surgery in large animals as a direct visual guidance throughout the entire sentinel lymph node mapping procedure, which permits a real-time confirmation of complete resection. Capped InP@ZnS QDs were used for cellular imaging by Kobayashi et al $^{12}$ in order to simultaneously visualize the draining of five separate lymphatic flows.

Heavy metal-free/cadmium-free biocompatible QDs with a good photoluminescence quantum yield were recently evaluated for their capability for lymph node mapping with the aid of an ex vivo imaging model. These indium-based QDs showed a strong retention confined to the regional lymph, fast localization (minutes) and stable photoluminescence. Moreover, the lack of any Class A elements $(\mathrm{Cd}, \mathrm{Hg}$ and $\mathrm{Pb})$ in their structure makes suitable candidates for future biomedical applications. ${ }^{13}$ Another interesting approach for biomedical imaging applications was reported by $\mathrm{Yu}$ et al, which developed gadolinium-doped carbon dots for fluorescence/magnetic resonance imaging (MRI) imaging. A one-pot hydrothermal method was employed in order to obtain bio-imaging nanoprobes $(-5.5 \mathrm{~nm}$ in size, nearly spherical) from citric acid, with high spatial resolution for MRI and enhanced fluorescence compared to that of carbon dots alone. ${ }^{14}$
Nitrogen and sulfur co-doped carbon dots (N,S/CDs), $1.7 \mathrm{~nm}$ in size, with a high fluorescence quantum yield (39.7\%) were obtained by Chen et al. They posed low toxicity and good biocompatibility when co-incubated with HeLa cells. These types of doped CDs can be used to detect $\mathrm{Cr}$ (IV) by means of fluorescence quenching. ${ }^{15}$ In another case, gadopentetic acid was used as the Gd source and glycine as the surface passivation agent in order to obtain Gd-doped carbon dots with an average size of $\sim 18 \mathrm{~nm}$ capable of enhancing irradiation dose deposition. ${ }^{16}$

The work conducted by Han et al showed that antibodyconjugated QDs can diffuse into the bone marrow and label single cells of rare populations of progenitor and hematopoietic cells. By employing this method, the disadvantages associated with fluorescently labeled antibodies such as low photo-stability, small multiphoton action cross-section and asymmetric emission are no longer an issue. ${ }^{17}$

In order to achieve superior biomedical properties, Osifeko and Nyokong have proposed hybrid nanoparticles with phthalocyanines in combination with QDs, magnetic nanoparticles and gold nanoparticles (GNPs). Their results showed an increase in triplet quantum yields for phthalocyanines in the presence of QDs and GNPs, while a general decrease in fluorescence quantum yields for them was observed in the presence of all the nanoparticles investigated (QDs, GNPs and magnetic nanoparticles). ${ }^{18}$ Glutathione-capped FePt-CdS bimodal nanoprobes were proposed by Jha et al for fluorescence imaging and MRI. The synthesis route described thioglycolic acid-capped FePt magnetic nanoparticles used as templates for the development of the CdS QD fluorescent counterparts. In this case, the glutathione coating provided biocompatibility and ligand attachment sites on the nanoprobes. ${ }^{19}$

Core-shell QDs such as CdSe@ZnS were employed in the quantitative determination of micrococcal nuclease, the extracellular nuclease of Staphylococcus aureus. In this case, the QDs were conjugated to a single-stranded DNA (ssDNA)-fluorescent dye FRET probe. ${ }^{20}$ Hafian et al undertook multiphoton imaging of tumor biomarkers by conjugating single-domain antibodies to CdSe@ZnS QDs. These nanoprobes, with a hydrodynamic diameter of $11.9 \mathrm{~nm}$, offer a viable alternative to conjugates of conventional organic fluorophores for imaging biomarkers. ${ }^{21}$ Yakoubi et al focused on obtaining core-shell $\mathrm{Cu}$-doped $\mathrm{CdZnS}$ QDs via a low-temperature aqueous-based route.Their Cu:CdZnS@ ZnS QDs presented high stability and high photoluminescence quantum efficiency, with the option to tune the PL from 470 to $620 \mathrm{~nm}$ by increasing $\mathrm{Cd}$ and $\mathrm{Cu}^{2+}$ composition. ${ }^{22}$ 


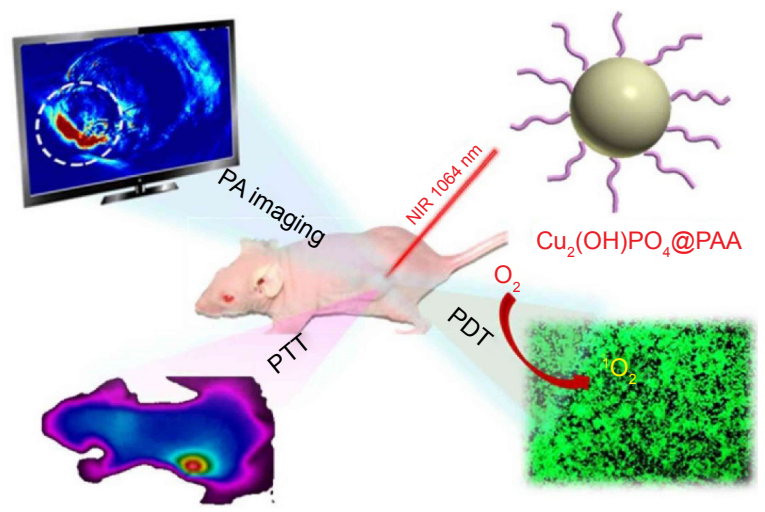

Figure I Proposed $\mathrm{Cu}_{2}(\mathrm{OH}) \mathrm{PO}_{4} @ P A A$ "three-in-one” multifunctional theranostic platform.

Notes: Reprinted with permission from Guo W, Qiu Z, Guo C, et al. Multifunctional theranostic agent of $\mathrm{Cu} 2(\mathrm{OH}) \mathrm{PO} 4$ quantum dots for photoacoustic image-guided photothermal/photodynamic combination cancer therapy. ACS Appl Mater Interfaces. 2017;9(II):9348-9358. Copyright 2017 American Chemical Society. ${ }^{23}$

Abbreviations: $\mathrm{CU}_{2}(\mathrm{OH}) \mathrm{PO}_{4} @ P A A$, polyacrylic acid-coated $\mathrm{Cu}_{2}(\mathrm{OH}) \mathrm{PO}_{4}$ quantum dots; PA, photoacoustic; PDT, photodynamic therapy; PTT, photothermal therapy.

The work of Guo et al focused on the fabrication of polyacrylic acid-coated $\mathrm{Cu}_{2}(\mathrm{OH}) \mathrm{PO}_{4}$ quantum dots $\left(\mathrm{Cu}_{2}(\mathrm{OH})\right.$ $\mathrm{PO}_{4} @$ PAA QDs) that exhibit a strong NIR photo-absorptive ability as a "three-in-one" multipurpose cancer theranosic system (Figure 1). A combination phototherapy of photodynamic therapy (PDT) and photothermal therapy (PTT) along with photoacoustic imaging modality was achieved. Under NIR excitation, a local hyperthermia and reactive oxygen species (ROS) were generated realizing the synergetic PTT/PDT therapeutic effects. Also these QDs presented themselves as good candidates for in vivo photoacoustic imaging on the tumor site for realizing an imaging-guided therapeutic process. ${ }^{23}$ Iodine-doped carbon dots (I-doped CDs), monodispersed spherical nanoparticles $\sim 2.7 \mathrm{~nm}$ in diameter, were synthesized by Zhang et al in order to be used as computed tomography contrast agents and fluorescence probes. These I-doped CDs displayed wavelength-dependent excitation and stable photoluminescence, superior X-ray attenuation properties in vitro and good biocompatibility. ${ }^{24}$

\section{Drug delivery}

The development of diagnostic and treatment capabilities into one nanoparticle-based agent has been the focus of several groups. QDs are good candidates as theranostic platforms, as they can act as the main nanocarrier or be part of a more complex architecture as the fluorescent labels.

Paclitaxel (PTX), a widely acknowledged drug choice for the treatment of various human cancers, along with CdTe@ CdS@ZnS QDs were co-loaded in nanostructured lipid carriers in order to have theranostic approach in cancer therapy.
The results from Olerile et $\mathrm{al}^{25}$ showed an encapsulation efficacy of $\sim 80 \%$ and drug loading of $4.68 \%$ with a tumor growth inhibition rate of $77.85 \%$. In another study, hybrid silica nanocapsules that were loaded with $\mathrm{ZnSe}: \mathrm{Mn} / \mathrm{ZnS}$ core-shell and the anticancer drug PTX have been proposed by Zhao et al as theranostic platforms for chemotherapy and fluorescence imaging. The setup involved hydrophobic inner cores that contain the PTX molecules and the ZnSe:Mn@ZnS QDs and a hydrophilic silica outer shell with amino groups on the surface as anchors for the targeting molecules. Results showed that solubility of PTX was enhanced 630 times while the drug could be sustained released in $12 \mathrm{~h} .{ }^{26}$

Doxorubicin (DOX) was loaded on to $\mathrm{pH}$-responsive ZnO QDs by Cai et al. They synthetized ultrasmall QDs $(\sim 3 \mathrm{~nm})$ functionalized with poly(ethylene glycol) (PEG) and hyaluronic acid to target the overexpressed glycoprotein CD44 in cancer cells and DOX as the model drug for the study. This drug delivery platform for intracellular controlled release of drugs could release its DOX payload under the acidic intracellular conditions (Figure 2) ${ }^{27}$ The work of Yang et al focused on quercetin (QE)-loaded CdSe@ZnS QDs as anticancer and antibacterial nanoplatforms. Their results showed that QE-loaded CdSe@ZnS were more effective against drug-resistant Escherichia coli and Bacillus subtilis and then QE or CdSe nanoparticles alone. The anticancer activity assay was focused on the proliferation and migration of BGC-823 cells, which showed an increase in cytotoxicity of two- to sixfold compared to raw $\mathrm{QE}$ and CdSe QDs. ${ }^{28}$

For the enhanced targeted delivery of 5-fluorouracil (5-FU), the model proposed by Bwatanglang et al made use of folate receptors expressed in malignant tissues. $\mathrm{Mn}: \mathrm{ZnS}$ QDs were encapsulated in chitosan (CS) biopolymer and functionalized with folic acid (FA). The resulted composite (5-FU@FACS-Mn:ZnS) was assessed in vivo in tumor-bearing mice, where they contributed to smaller tumor size and less events of metastasis in the lungs compared to the groups treated with just the 5-FU drug. The in vitro results were also encouraging; the 5-FU@FACS$\mathrm{Mn}: \mathrm{ZnS}$ NPs induced higher levels of apoptosis in breast cancer cells (MDA-MB231) compared to the effect of just the 5-FU drug toward the same cell line. ${ }^{29}$ Gemcitabine (GEM)-programed nanovectors for targeted pancreatic cancer therapy were obtained by functionalizing $\mathrm{CdSe} / \mathrm{ZnS}$ QDs with matrix metalloproteinase-9 (MMP-9) detachable, PEG, cathepsin B cleavable GEM and targeting ligand CycloRGD. In this case, the PEG corona ensured prolonged blood circulation time and avoided nonspecific interactions. Once the QDs are accumulated in the tumor, the corona is 


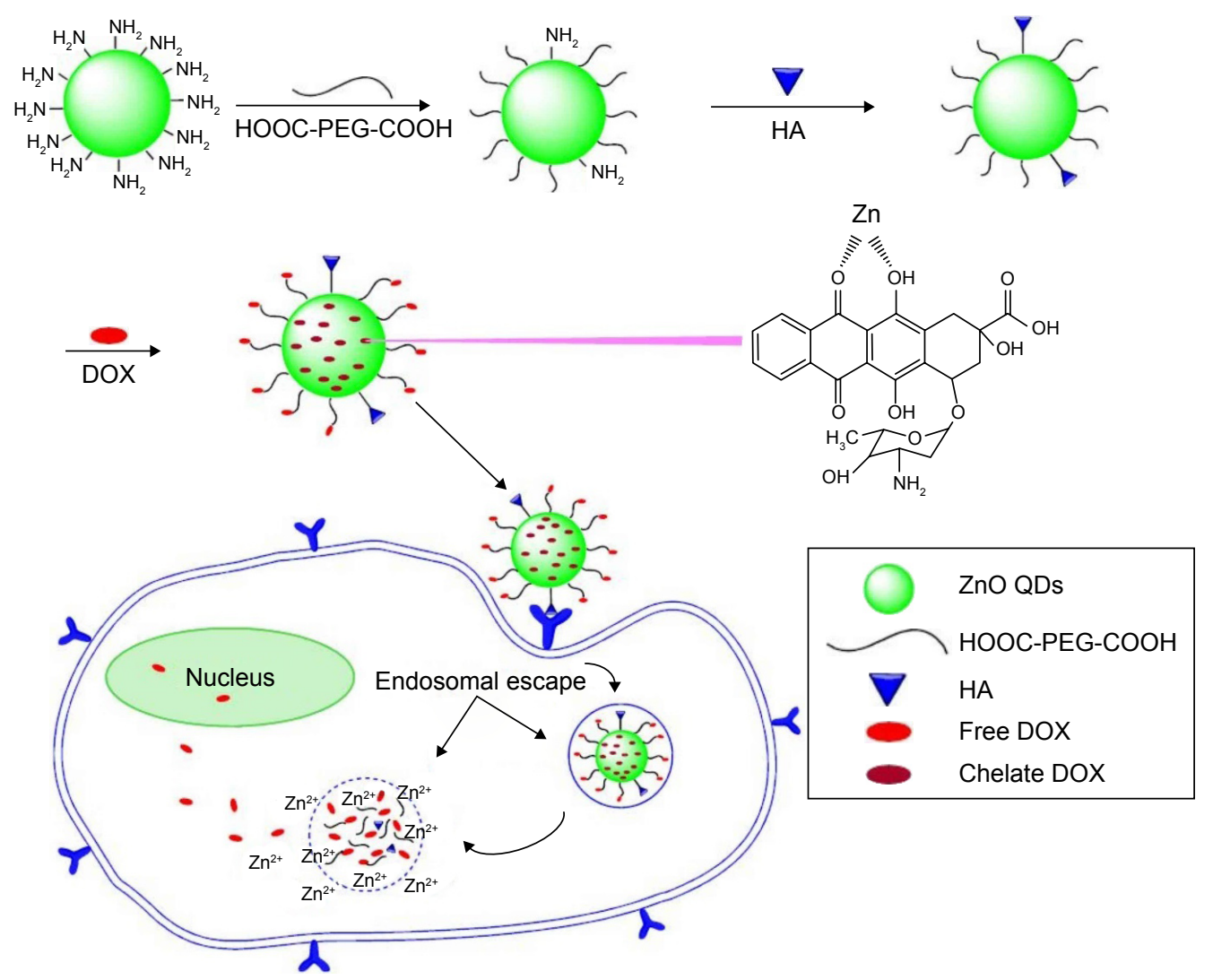

Figure 2 Graphical representation of the hyaluronic acid-ZnO quantum dots-dicarboxyl-terminated poly(ethylene glycol) (HA-ZnO-PEG) drug delivery system. Notes: Reprinted with permission from Cai X, Luo Y, Zhang W, et al. pH-Sensitive ZnO quantum dots-doxorubicin nanoparticles for lung cancer targeted drug delivery. ACS Appl Mater Interfaces. 2016;8(34):22442-22450. Copyright 2016 American Chemical Society. ${ }^{27}$

Abbreviations: DOX, doxorubicin; HA, hyaluronic acid; PEG, poly(ethylene glycol); QDs, quantum dots.

removed by the overexpressed MMP-9 and the RGD exposed in order to enhance the cellular internalization process. Inside the pancreatic cells, elevated levels of lysosomal cathepsin B promote the release of GEM; a schematic illustration of the process is presented in Figure $3 .{ }^{30}$

Chiu et al developed a nanotheranostic platform based on carbon quantum dots (CQDs) doped with $\mathrm{S}, \mathrm{N}$ and $\mathrm{Gd}$ (GdNS@CQDs). In order to have a targeted dual mode fluorescence/MRI, the GdNS@CQDswere functionalized with FA through ECD/Sulfo-NHS reaction and the targeting capability was evaluated on two cancerous cell lines, HeLa and HepG2. The obtained nanoplatforms showed good stability, biocompatibility and low toxicity. Moreover, the drug-loading capacity for FA-GdNS@CQDs with DOX, an anticancer drug, was $\sim 80 \%$ and its release proved to be $\mathrm{pH}$-sensitive. Thus, these nanotheranostic platforms have been reported to have bimodal contrast imaging functionality (fluorescence/magnetic resonance), cancer cell targeting and pH-sensitive drug release capabilities. ${ }^{31}$

Unlike semiconductor QDs, CQDs have low toxicity, good biocompatibility and broad excitation spectra. CQDs from sodium alginate for gene delivery applications have been developed by Zhou et al in a one-step green process. In the proposed one-step hydrothermal carbonization route, alginate is both the carbon source and the cationization agent. The obtained CQDs, with their strong capacity to condense plasmid DNA and high transfection efficiency, could play a dual role as gene vectors and as bioimaging probes. ${ }^{32}$ Graphene quantum dots (GQDs) were shown to enhance the anticancer activity of cisplatin by Sui et al, mainly through the fact that they enhance cellular uptake of the drug; the proposed mechanism is depicted by Sui et al..$^{33}$

Another exciting application for GQDs has been reported in the fight against Alzheimer's disease, where glycine-prolineglutamate-conjugated graphene quantum dots (GQDGs) were developed and evaluated on in vitro and an in vivo murine model. The GQDGs had an inhibitory effect on the aggregation of amyloid- $\beta$ fibrils, the number of newly generated neuronal precursor cells and neurons increased. ${ }^{34}$

\section{Sensors}

By employing the unique properties of QDs, new strategies for the identification and quantification of biological relevant molecules have been presented in the last years. 

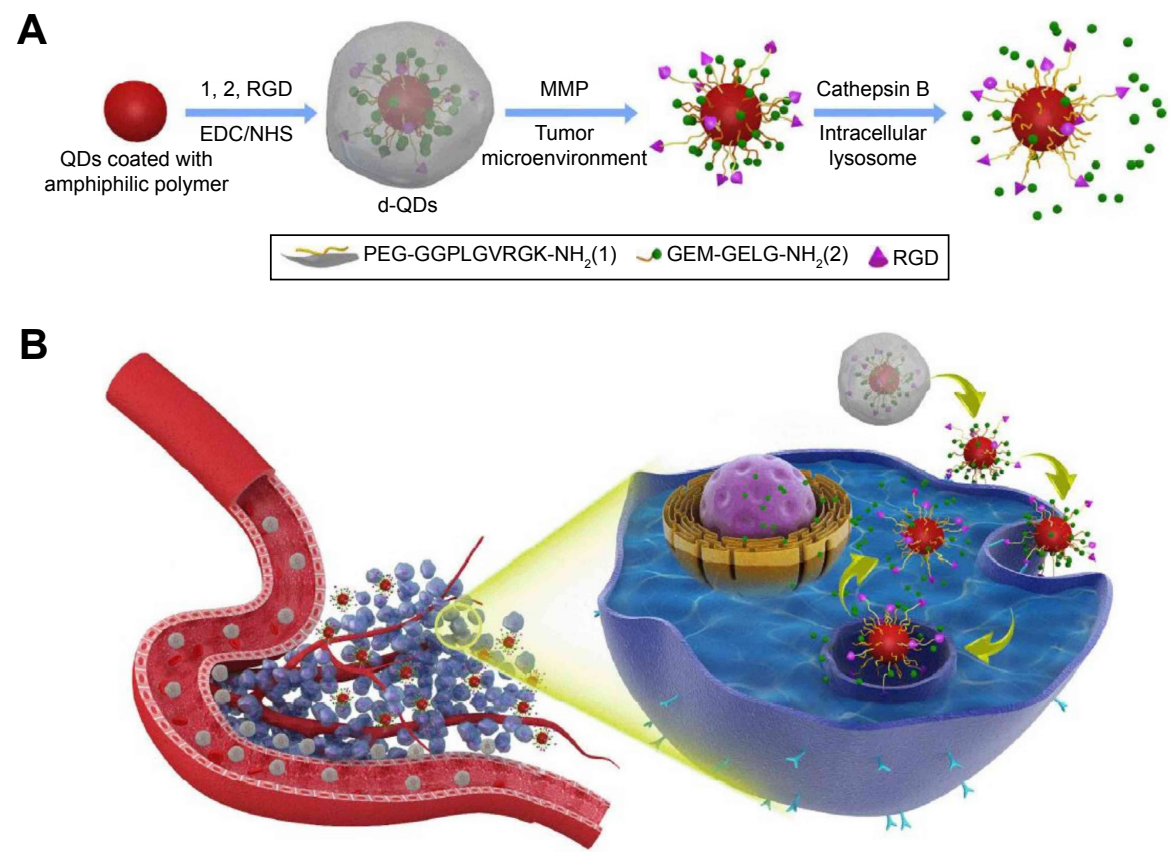

Figure 3 (A) Schematic illustration of the nanovectors preparation protocol and their enzyme sensitive behavior. (B) Schematic illustration of the nanovectors delivering GEM to pancreatic cancer cells.

Notes: Reprinted with permission from Han H, Valdeperez D, Jin Q, et al. Dual enzymatic reaction-assisted gemcitabine delivery systems for programmed pancreatic cancer therapy. ACS Nano. 2017; I (2):I28I-1291. Copyright 2017 American Chemical Society. ${ }^{30}$

Abbreviations: d-QDs, dual-enzyme quantum dots; EDC, I-Ethyl-3-(3-dimethylaminopropyl)carbodiimide; GEM, gemcitabine; MMP, matrix metalloproteinase; NHS, N-hydroxysuccinimide; RGD, CycloRG; QDs, quantum dots.

One of the first reports of QDs $(\mathrm{ZnS} @ \mathrm{CdSe})$ for ultrasensitive nonisotopic detection was published by Chan and Nie. ${ }^{35}$ For the detection of the CA-125 serum biomarker in patients with ovarian cancer, Johari-Ahar et al proposed an impedimetric immunosensor that consisted of a mercaptopropionic acidmodified gold electrode functionalized with silica-coated gold nanoparticles, CdSe QDs and the anti-CA-125 monoclonal antibody. This setup allowed for the detection of the targeted biomarker down to $0.0016 \mathrm{U} / \mathrm{mL}^{36}$

Leishmaniosis is a parasitic disease, one of the six most important diseases that affects humans and various animal species. Andreadou et al developed a Leishmania-specific surface antigens detection method based on a combination of magnetic beads and CdSe QDs with a specificity of $100 \%$ and a low limit of detection of 3,125 ng/ $\mu \mathrm{L}$ for Leishmania DNA. The method was based on the fact that the analytes were isolated from the solutions with the aid of the magnetic beads and the detection part was played by the QDs. ${ }^{37}$ A sensitive sensor for probing the interaction of clofazimine (an effective drug multidrug-resistant tuberculosis and breast cancer) with proteins with the aid of fluorescence spectroscopic techniques was proposed by Yang et al. They developed $\mathrm{CdZnSeS} / \mathrm{ZnS}$ alloyed core/thick-shell QDs as energy donors Förster resonance energy transfer (FRET) applications (Figure 4). These QDs were capped with multifunctional polymer ligands containing dihydrolipoic acid via a direct ligand-exchange method and further functionalized with cyanine 3-labeled human serum albumin. ${ }^{38}$

$\mathrm{Zn}^{2+}$-modulated L-cysteine-capped CdTe QDs were used for the detection of adenosine $5^{\prime}$-triphosphate (ATP) in human serum samples. In this case, the $\mathrm{Zn}^{2+}$ ions can bind with the ATP molecules through $\mathrm{Zn}-\mathrm{O}-\mathrm{P}$ bonds, and the initial quenching process resulted from the interaction of $\mathrm{Zn}^{2+}$ ions with the L-cysteine layer from the CdTe QDs would be avoided. ${ }^{39}$

Another application of CdTe QDs has been proposed in combination with the immunomagnetic separation technique for rapid enumeration of E. coli. For this, magnetic $\mathrm{Fe}_{3} \mathrm{O}_{4} @$ Au nanoparticles were functionalized with biotinylated E. coli-specific antibodies, while CS was used to modify the CdTe QDs. By using magnetic nanoparticles for bacteria separation and QDs for labeling, the method achieves a detection limit of $30 \mathrm{cfu} / \mathrm{mL}^{-1}$ of E. coli and a high specificity. ${ }^{40}$

In order to investigate heme iron absorption, Geng et al proposed using hemin-conjugated $\mathrm{CdSe} @ \mathrm{ZnS}$ nanoprobes in in vitro and in vivo experimental setups. Their results showed that this QD configuration is a suitable tracer for the heme iron absorption and that both active transport hemoglobin carrier protein-1 and endocytosis pathways are involved in heme uptake in Caco-2 cells. ${ }^{41}$ In another approach, core/ multishell QDs (CdSe@ZnS@CdS@ZnS) with photoluminescence quantum yields as high as $100 \%$ were obtained by layer-by-layer precursor deposition. In this case, each layer 


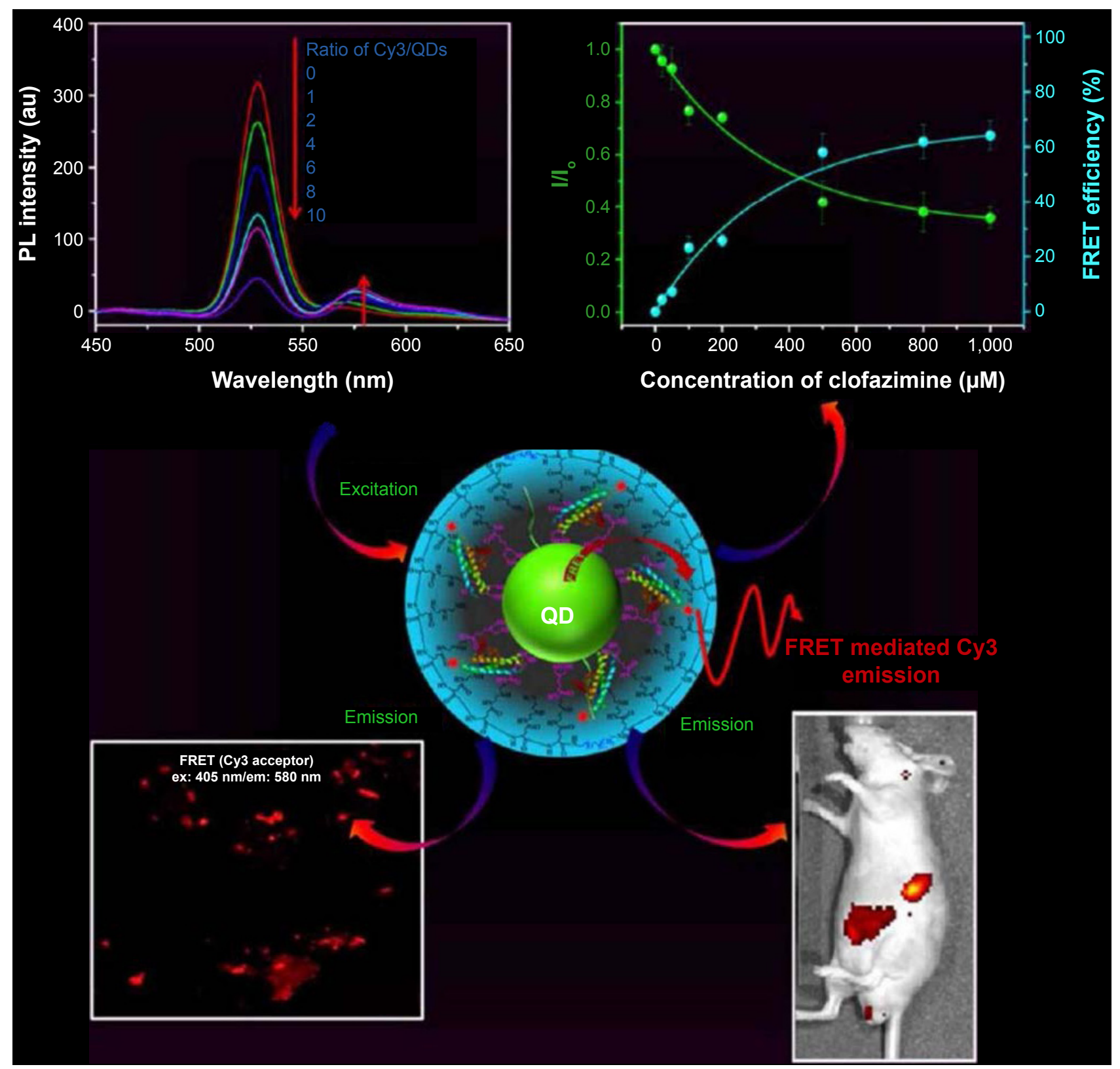

Figure $4 \mathrm{CdZnSeS/ZnS} \mathrm{alloyed} \mathrm{core/thick-shell} \mathrm{QDs} \mathrm{as} \mathrm{energy} \mathrm{donors} \mathrm{for} \mathrm{FRET} \mathrm{applications.}$

Notes: Adapted with permission from Yang HY, Fu Y, Jang MS, et al. Multifunctional polymer ligand interface CdZnSeS/ZnS quantum Dot/Cy3-labeled protein pairs as sensitive FRET sensors. ACS Appl Mater Interfaces. 2016;8(51):35021-35032. Copyright @ 2016 American Chemical Society. ${ }^{38}$

Abbreviations: Cy3, cyanine 3; em, emission; ex, excitation; FRET, Förster resonance energy transfer; PL, photoluminescence; QD, quantum dot.

deposited on to the CdSe core had a specific role: the first $\mathrm{ZnS}$ shell acts as a potential barrier for electron-hole pairs, the CdS shell is a separator between the two $\mathrm{ZnS}$ shells, increasing shell thickness, and the outer $\mathrm{ZnS}$ shell is an additional potential barrier, compatible with already known protocols for QD functionalization. ${ }^{42}$ A QD aggregationbased detection strategy to quantify heparin was proposed by Dong et al, where antithrombin III (AT III)-labeled QDs specifically bind to active heparin molecules to form complexes that aggregate upon the addition of cationic surfactant micelles (Figure 5). This approach had a linearity in the range of $4.65 \times 10^{-4} \mathrm{U} / \mathrm{mL}$ to $0.023 \mathrm{U} / \mathrm{mL}$ and a limit of detection of $9.3 \times 10^{-5} \mathrm{U} / \mathrm{mL} .{ }^{43}$

The specific recognition of transferrin (TRF), a $77 \mathrm{kDa}$ glycoprotein responsible for iron transport, was achieved by Chang et al by using mercaptophenylboronic acid-capped Mn-doped ZnS QDs (QDs-MBA). The method is based on a turn-on effect by the fact that TRF binds to the MBA via boronate affinity interaction to efficiently enhance the fluorescence of the QD@MBA, with a limit of detection 

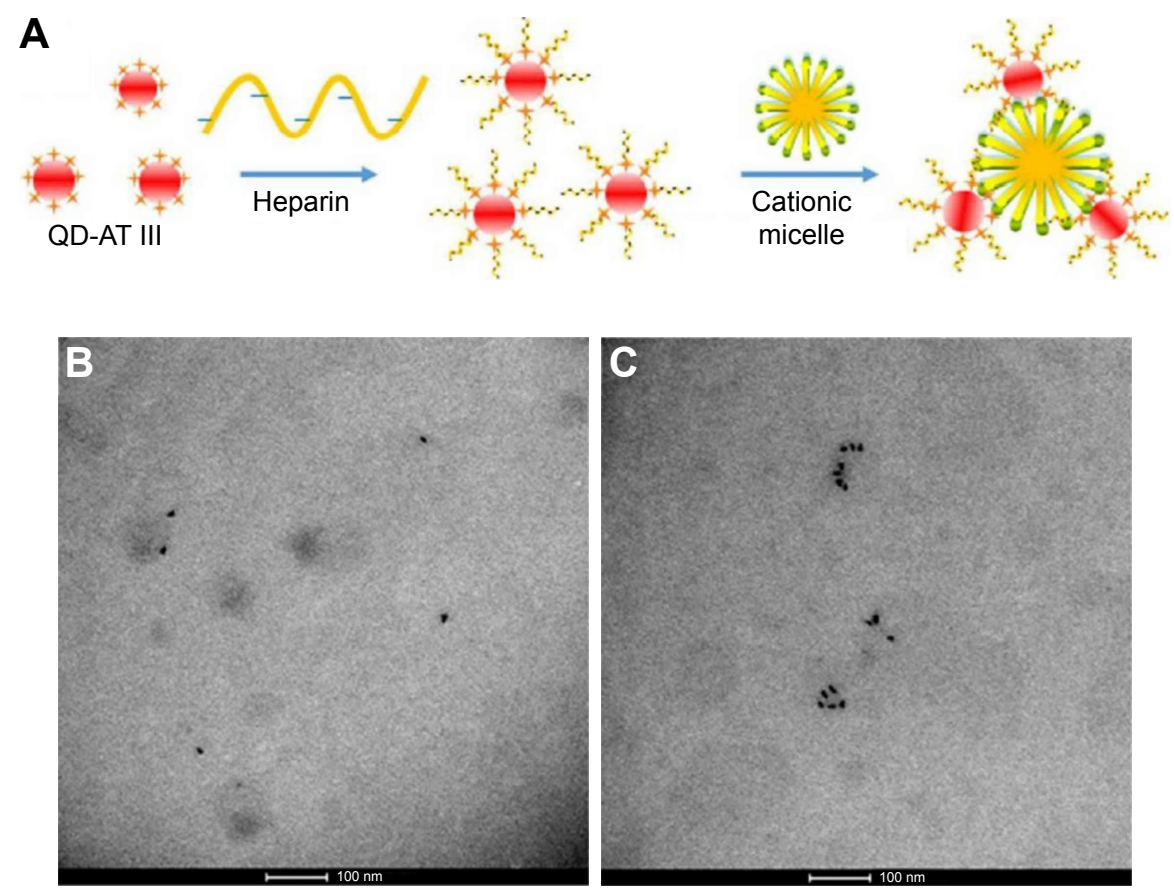

Figure 5 (A) Scheme of aggregated QD formation. (B) TEM image of QD-AT III-heparin without CTAB. (C) TEM image of QD aggregation with CTAB.

Notes: Reprinted with permission from Dong S, Liu X, Zhang Q, et al. Sensing active heparin by counting aggregated quantum dots at single-particle level. ACS Sens. 2017;2(1):80-86. Copyright 2017 American Chemical Society. ${ }^{43}$

Abbreviations: AT III, antithrombin III; CTAB, cetyl trimethylammonium bromide; QD, quantum dot; TEM, transmission electron microscopy.

for TRF down to $5.69 \times 10^{-9} \mathrm{M}$ and a serum sample recovery from $87.9 \%$ to $96.5 \%$. This approach can be employed for the detection of trace glycoproteins in complex biological samples. ${ }^{44}$

Graphene quantum dots (GQDs) present an interesting approach for L-ascorbic acid detection and alkaline phosphatase (ALP) activity. Huang et al demonstrated that GQDs can act as sensors, their fluorescence quenched in the presence of para-benzoquinone $(\mathrm{BQ})$ and then turned on when L-ascorbic acid reduces the BQ molecules. For ALP activity evaluation, the ALP hydrolyzed L-ascorbic acid-2-phosphate and produced ascorbic acid, which turned on the fluorescence of the nanoprobes. ${ }^{45}$

Caballero-Diaz et a ${ }^{46}$ proposed using nitrogen-doped grapheme quantum dots (N-GQDs) as the fluorescent probe along with acethylcholinesterase (AChE) enzyme as the biorecognition element for the detection of fenoxycarb pesticide.

For the detection of $\mathrm{Pb}^{2+}$ ions, carbon dots (CDs) obtained through a green synthesis method from Ocimum sanctum leaves was proposed by Kumar et al. CDs, $4-7 \mathrm{~nm}$ in size, were fabricated in a one-step hydrothermal method from the natural source with no supplementary functionalization steps needed in order to use them for lead sensing and cell imaging. The detection of $\mathrm{Pb}^{2+}$ was based on the fact that the lead ions induce fluorescence quenching of the CDs with a linear detection range of $0.01-1 \mu \mathrm{M}$ and a limit of detection of $0.59 \mathrm{nM}^{47}$

\section{Toxicity}

The toxicity concerns regarding QDs are mainly related to their chemical composition, especially in the case of QD containing heavy metal ions such as $\mathrm{Cd}$ and $\mathrm{Hg}$. In order to use QDs in clinical applications, they must exhibit minimal toxicity. ${ }^{48}$ This can be ensured by functionalizing the QD surface with biocompatible molecules. One approach for QD functionalization is to take advantage of the fact that mercapto groups can bind easily to zinc atoms and small cysteine-rich proteins that can bind up to monovalent or up to seven divalent heavy metal ions. ${ }^{49}$ Tmejova et al investigated the interactions between mercaptosuccinic capped CdTe (CdTe-MSA) QDs and metallotionein (MT) proteins. Their work showed that, along with electrostatic forces, QD size has a strong influence on MT proteins and QDs, with the smallest QDs having a higher affinity for this type of interaction. ${ }^{50}$

CdTe QDs were used as a model for investigating the effects of QDs on the nuclear factor- $\kappa \mathrm{B}(\mathrm{NF}-\mathrm{\kappa B})$ signaling pathway in in vitro and in vivo experiments. In this case, it was found that QDs can selectively inhibit both canonical and the non-canonical NF- $\kappa B$ activation, which can lead to new 
directions in anti-cancer, anti-inflammatory and anti-viral drug therapies. ${ }^{51}$ In addition, it was found by $\mathrm{Wu}$ et al that CdTe QDs can alter the molecular basis of fatty acid synthesis and degradation, without influencing the degradation of phospholipids in the Caenorhabditis elegans animal model. The increased fat storage in the intestines of the nematodes was attributed to the prolonged defection cycle length and not to the cadmium ions released from the QDs. These findings shed light on the environmental impact of CdTe QDs on the lipid metabolism in certain animals. ${ }^{52}$

The work conducted by Fan et al focused on CdTe@CdS QDs toxicity in in vitro and in vivo experimental setups. QD-induced nanotoxicity was observed in the studied cell lines, with autophagy as the general response to QD exposure. For the murine model, the injection of QDs determined splenic injury, liver injury, nephrotoxicity and hematopoietic disorders, with ROS involved in toxicity induction and autophagy initiation. ${ }^{53}$

Cadmium oxide nanoparticle toxicity was evaluated by Balmuri et al on a zebrafish model. They studied two types of $\mathrm{CdO}$ nanoparticles: one where $\mathrm{CdO}$ was obtained through calcination of $\mathrm{Cd}(\mathrm{OH})_{2}$ to yield nanoparticles without any organic molecules on the surface (CdO-1) and another type that involved the calcination of $\mathrm{Cd}$-citrate (CdO-2). The toxic effect registered for the $\mathrm{CdO}-2$ was lower than for the CdO-1; this result could be attributed to a carbon layer that covered the surface of $\mathrm{CdO}-2$ during the calcination process. Thus, by using organic molecules in the preparation of $\mathrm{CdO}$ nanoparticles, their toxicity can be reduced by forming carbon surface coverage that impedes the release of $\mathrm{Cd}^{2+}$ ions in biological systems. ${ }^{54}$

Saccharomyces cerevisiae yeast was used, as a model, by Pasquali et al in order to shed light on the effect of $\mathrm{CdS}$ QDs on the complex genetic networks that regulate nucleomitochondrial interaction. The results showed the disruption of the mitochondrial morphology, a reduced mitochondrial function and induced oxidative stress. ${ }^{55}$ The in vivo and in vitro assessment of nanotoxicity of CS-functionalized CdS QDs was reported by de Carvalho et al. Results showed that even when CdS QDs were coated with this biocompatible aminopropylsaccharide shell induced a severe inhibition of cell viability for human osteosarcoma cells (SAOS) and for human embryotic kidney cells (HEK293T). However, for the in vivo experiments, BALB/c mice were intravenously injected with the CS-coated CdS QDs where they showed no evident toxicity after 30 days. ${ }^{56}$ Blood clearance, distribution,

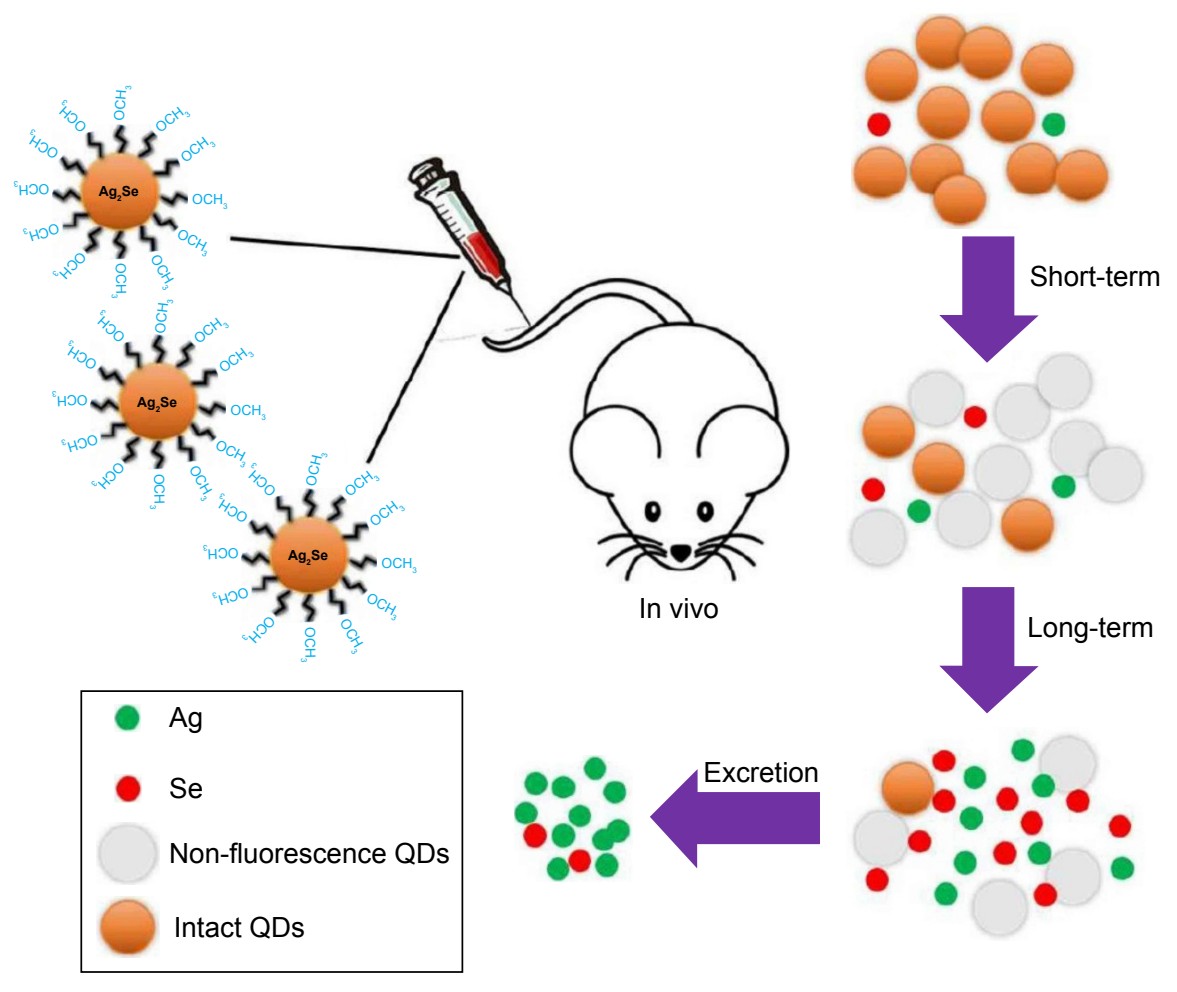

Figure 6 Biotransformation of Ag2Se, where Ag released from the QDs is excreted through feces and urine and the Se element is hardly excreted. Notes: Reprinted with permission from Tang H, Yang ST, Ke DM, et al. Blood clearance, distribution, transformation, excretion, and toxicity of near-infrared quantum dots Ag2Se in mice. ACS Appl Mater Interfaces. 2016;8(28):17859-17869. Copyright 2016 American Chemical Society. ${ }^{57}$

Abbreviation: QDs, quantum dots. 
transformation, excretion and toxicity of PEG-coated $\mathrm{Ag}_{2} \mathrm{Se}$ QDs (fluorescent probes in the second NIR window) in mice after intravenous was assessed by Tang et al. They found that $\mathrm{Ag}_{2} \mathrm{Se}$ QDs-PEG prefer to accumulate in the spleen and liver but transformed and/or cleared within 1 day after administration. The released Ag from the QDs is excreted through both feces and urine, and the Se element is hardly excreted (Figure 6). ${ }^{57}$

In order to passivate QDs for biological applications. They can be encapsulated in phospholipid micelles. This approach has several advantages, such as it does not alter their surface, the optical properties of the QDs are retained, due to the highly dense surface of the micelles nonspecific adsorption is prevented and the supramolecular architecture is maintained by local hydrophobic interactions. ${ }^{58}$ Other functionalization strategies in order to confer biological activity and low toxicity to QDs is to conjugate them to antibodies. For example, a QD with a $4 \mathrm{~nm}$ diameter can have its surface functionalized with 2-5 protein molecules or 50 small molecules. ${ }^{59}$ There are a number of available methods ranging from noncovalent binding (electrostatic interactions ${ }^{60}$ or biotin-avidin systems ${ }^{4}$ ) to covalent binding (between amine and carboxylic acid groups, between aldehyde and hydrazine functions and between amino and sulfhydryl groups ${ }^{61}$ ). The potential toxicity of different types of QDs such as metal-free or CQDs remains one of the main interests of the scientific community with the need of better understanding the interactions between QDs and biological macromolecules.

\section{Conclusion}

It is clear that QDs have great potential for applications in areas such as drug delivery, sensors and bio-imaging. In order to see QDs realistically translated in clinical applications, several issues still need to be addressed, such as overall toxicity, body clearance, synthesis protocol scalability, environmental impact, manufacturing costs and so on. By combining QDs with other types of nanoparticles and/or biological active molecules, theranostic platforms are constantly being developed. This review summarizes the latest developments available in literature regarding the use of QDs for medical applications.

\section{Acknowledgments}

This work was supported by the Romanian National Authority for Scientific Research and Innovation, CNCSUEFISCDI, project numbers PN-II-RU-TE-2014-4-2267, PN-II-PT-PCCA-2013-4-1553, PN-III-P2-2.1-BG-20160446 and PN-III-P2-2.1-PED-2016-1742.

\section{Disclosure}

The authors report no conflicts of interest in this work.

\section{References}

1. Ekimov A, Onushenko AA. The quantum size effect in three-dimensional microscopic semiconductor. JETP Lett. 1981;34:363-366.

2. Rocha TL, Mestre NC, Saboia-Morais SMT, Bebianno MJ. Environmental behaviour and ecotoxicity of quantum dots at various trophic levels: a review. Environ Int. 2017;98:1-17.

3. Volkov Y. Quantum dots in nanomedicine: recent trends, advances and unresolvedissues. Biochem Biophys Res Commun. 2015;468(3): 419-427.

4. Xing SG, Xiong QR, Zhong Q, et al. Recent research advances of antibody-conjugated quantum dots. Chin J Anal Chem. 2013;41: 949-955.

5. Ji X, Pen F, Zhong Y, Su Y, He Y. Fluorescent quantum dots: synthesis, biomedical optical imaging, and biosafety assessment. Colloids Surf B Biointerfaces. 2014;124:132-139.

6. Marukhyan SS, Gasparyan VK. Fluorometric immunoassay for human serum albumin based on its inhibitory effect on the immunoaggregation of quantum dots with silver nanoparticles. Spectrochim Acta A Mol Biomol Spectrosc. 2017;173:34-38.

7. Karakoti AS, Shukla R, Shanker R, Singh S. Surface functionalization of quantum dots for biological applications. Adv Colloid Interface Sci. 2015;215:28-45.

8. Zharkova IR, Markina NE, Markin AV, Drozd DD, Speranska ES, Goryacheva IY. Influence of electric field ontheproperties of the polymer stabilized luminescent quantum dots in aqueous solutions. J Luminescence. 2016;176:65-70.

9. Smith AM, Duan H, Mohs AM, Nie S. Bioconjugated quantum dots for in vivo molecular and cellular imaging. Adv Drug Deliv Rev. 2008; 60(11):1226-1240.

10. Weissleder R. A clearer vision for in vivo imaging: progress continues in the development of smaller, more penetrable probes for biological imaging. Nat Biotechnol. 2001;19(4):316-317.

11. Kim S, Lim YT, Soltesz EG, et al. Near-infrared fluorescent type II quantum dots for sentinel lymph node mapping. Nat Biotechnol. 2004;22(1):93-97.

12. Kobayashi H, Hama Y, Koyama Y, et al. Simultaneous multicolor imaging of five different lymphatic basins using quantum dots. Nano Lett. 2007;7(6):1711-1716.

13. Yaghini E, Turner HD, LeMarois AM, Suhling K, Naasani I, MacRobert AJ. In vivo biodistribution studies and ex vivo lymph node imaging using heavy metal-free quantum dots. Biomaterials. 2016;104: 182-191.

14. Yu C, Xuan T, Chen Y, et al. Gadolinium-doped carbon dots with high quantum yield as an effective fluorescence and magnetic resonance bimodal imaging probe. J Alloys Comp. 2016;688:611-619.

15. Chen J, Liu J, Li J, Xu L, Qiao Y. One-pot synthesis of nitrogen and sulphur co-doped carbon dots and its application for sensor and multicolor cellular imaging. J Colloid Interface Sci. 2017;485:167-174.

16. Du F, Zhang L, Zhang L, et al. Engineered gadolinium-doped carbon dots for magnetic resonance imaging-guided radiotherapy of tumors. Biomaterials. 2017;121:109-120.

17. Han HS, Niemeyer E, Huang Y, et al. Quantum dot/antibody conjugates for in vivo cytometric imaging in mice. Proc Natl Acad Sci U SA. 2014; 112(5):1350-1355.

18. Osifeko O, Nyokong T. Synthesis and physicochemical properties of zinc and indium phthalocyanines conjugated to quantum dots, gold and magnetic nanoparticles. Dyes Pigm. 2016;131:186-200.

19. Jha DK, Saikia K, Chakrabarti S, et al. Direct one-pot synthesis of glutathione capped hydrophilic FePt-CdS nanoprobe for efficient bimodal imaging application. Mater Sci Eng C Mater Biol Appl. 2017;72: 415-424.

20. Huang S, Xiao Q, He ZK, et al. A high sensitive and specific QDs FRET bioprobe for MNase. Chem Commun. 2008;45:5990-5992. 
21. Hafian H, Sukhanova A, Turini M, et al. Multiphoton imaging of tumor biomarkers with conjugates of single-domain antibodies and quantum dots. Nanomedicine. 2014;10(8):1701-1709.

22. Yakouby A, Chaabane TB, Aboulaich A, et al. Aqueous synthesis of $\mathrm{Cu}$-doped CdZnS quantum dots with controlled and efficient photoluminescence. J Luminescence. 2016;175:193-202.

23. Guo W, Qiu Z, Guo C, et al. Multifunctional theranostic agent of $\mathrm{Cu} 2(\mathrm{OH})$ PO4 quantum dots for photoacoustic image-guided photothermal/ photodynamic combination cancer therapy. ACS Appl Mater Interfaces. 2017;9(11):9348-9358.

24. Zhang M, Ju H, Zhang L, et al. Engineering iodine-doped carbon dots as dual-modal probes for fluorescence and X-ray $\mathrm{CT}$ imaging. Int $J$ Nanomedicine. 2015;10(1):6943-6953.

25. Olerile LD, Liu Y, Zhang B, et al. Near-infrared mediated quantum dots and paclitaxel co-loaded nanostructured lipid carriers for cancer theragnostic. Colloids Surf B Biointerfaces. 2017;150:121-130.

26. Zhao T, Liu X, Li Y, et al. Fluorescence and drug loading proprieties of $\mathrm{ZnSe}: \mathrm{Mn} / \mathrm{ZnS}-\mathrm{Paclitaxel} / \mathrm{SiO} 2$ nanocapsules templated by $\mathrm{F} 127$ micelles. J Colloid Interface Sci. 2017;490:436-443.

27. Cai X, Luo Y, Zhang W, Du D, Lin Y. pH-Sensitive ZnO quantum dots-doxorubicin nanoparticles for lung cancer targeted drug delivery. ACS Appl Mater Interfaces. 2016;8(34):22442-22450.

28. Yang X, Zhang W, Zhao Z, et al. Quercetin loading CdSe/ZnS nanoparticles as efficient antibacterial and anticancer materials. $J$ Inorg Biochem. 2017;167:36-48.

29. Bwatanglang IB, Mohammad F, Yusof NA, et al. In vivo tumor targeting and anti-tumor effects of 5-fluororacil loaded, folic acid targeted quantum dot system. J Colloid Interface Sci. 2016;480:146-158.

30. Han H, Valdeperez D, Jin Q, et al. Dual enzymatic reaction-assisted gemcitabine delivery systems for programmed pancreatic cancer therapy. ACS Nano. 2017;11(2):1281-1291.

31. Chiu SH, Gedda G, Girma WM, et al. Rapid fabrication of carbon quantum dots as multifunctional nanovehicles for dual-modal targeted imaging and chemotherapy. Acta Biomater. 2016;46:151-164.

32. Zhou J, Deng W, Wang Y, et al. Cationic carbon quantum dots derived from alginate for gene delivery: one-step synthesis and cellular uptake. Acta Biomater. 2016;42:209-219.

33. Sui X, Luo C, Wang C, Zhang F, Zhang J, Guo S. Graphene quantum dots enhance anticancer activity of cisplatin via increasing its cellular and nuclear uptake. Nanomedicine. 2016;12(7):1997-2006.

34. Xiao S, Zhou D, Luan P, et al. Graphene quantum dots conjugated neuroprotective peptide improve learning and memory capability. Biomaterials. 2016;106:98-110.

35. Chan WCW, Nie S. Quantum dot bioconjugates for ultrasensitive nonisotopic detection. Science. 1998;281(5385):2016-2018.

36. Johari-Ahar M, Rashidi MR, Barar J, et al. An ultra-sensitive impedimetric immunosensor for detection of the serum oncomarker CA-125 in ovarian cancer patients. Nanoscale. 2015;7(8):3768-3769.

37. Andreadou M, Liandris E, Gazouli M, et al. Detection of Leishmaniaspecific DNA and surface antigens using a combination of functionalized magnetic beads and cadmium selenite quantum dots. J Microbiol Methods. 2016;123:62-67.

38. Yang HY, Fu Y, Jang MS, et al. Multifunctional polymer ligand interface $\mathrm{CdZnSeS/ZnS}$ quantum Dot/Cy3-labeled protein pairs as sensitive FRET sensors. ACS Appl Mater Interfaces. 2016;8(51):35021-35032.

39. Shi F, Li Y, Lin Z, Ma D, Su X. A novel fluorescent probe for adenosine 5-triphosphate detection based on $\mathrm{Zn}^{2+}$-modulated 1-cysteine capped CdTe quantum dots. Sens Actuators B Chem. 2015;220:433-440.

40. Dogan U, Kasap E, Cetin D, et al. Rapid detection of bacteria based on homogenous immunoassay using chitosan modified quantum dots. Sens Actuators B Chem. 2016;233:369-378.

41. Geng L, Duan X, Wang Y, et al. Quantum dots-hemin: preparation and application in the absorption of heme iron. Nanomedicine. 2016; 12(7):1747-1755.
42. Linkov P, Krivenkov V, Nabiev I, Samokhalov P. High quantum yield $\mathrm{CdSe} / \mathrm{ZnS} / \mathrm{CdS} / \mathrm{ZnS}$ multishell quantum dots for biosensing and optoelectronic applications. Mater Today. 2016;3(2):104-108.

43. Dong S, Liu X, Zhang Q, et al. Sensing active heparin by counting aggregated quantum dots at single-particle level. ACS Sens. 2017;2(1): $80-86$.

44. Chang L, He X, Chen L, Zhang Y. Mercaptophenylboronic acid-capped Mn-doped $\mathrm{ZnS}$ quantum dots for highly selective and sensitive fluorescence detection of glucoproteins. Sens Actuators B Chem. 2017;243: $72-77$.

45. Huang H, Wang B, Chen M, et al. Fluorescence turn-on sensing of ascorbic acid and alkaline phosphatase activity based on graphene quantum dots. Sens Actuators B Chem. 2016;235:356-361.

46. Caballero-Diaz E, Benitez-Martinez S, Valcarcel M. Rapid and simple nanosensor by combination of graphene quantum dots and enzymatic inhibition mechanisms. Sens Actuators B Chem. 2017;240:90-99.

47. Kumar A, CHowdhuri AR, Laha S, Mahto TK, Karmakar P, Sahu SK. Green synthesis of carbon dots from Ocimum sanctum for effective fluorescent sensing of $\mathrm{Pb}^{2+}$ ions and live cell imaging. Sens Actuators B Chem. 2017:242:679-686.

48. Park Y, Jeonga S, Kim S. Medically translatable quantum dots for biosensing and imaging. J Photochem Photobiol C Photochem Rev. 2017;30:51-70.

49. Krikova S, Fabrik I, Adam V, Hrabeta J, Eckschaler T, Kizek R. Metallothionein - a promising tool for cancer diagnostics. Bratislava Med J-Bratislavske Lekarske Listy. 2009;110:93-97.

50. Tmejova K, Hynek D, Kopel P, et al. Structural effects and nanoparticle size are essential for quantumdots-metallothionein complex formation. Colloids Surf B Biointerfaces. 2015;134:262-272.

51. Hu Z, Song B, Xu L, et al. Aqueous synthesized quantum dots interfere with the NF-kB pathway and confer anti-tumor, anti-viral and antiinflammatory effects. Biomaterials. 2016;108:187-196.

52. Wu Q, Zhi L, Qu Y, Wang D. Quantum dots increased fat storage in intestine of Caenorhabditis elegans by influencing molecular basis for fatty acid metabolism. Nanomedicine. 2016;12(5):1175-1184.

53. Fan J, Sun Y, Wang S, et al. Inhibition of autophagy overcomes the nanotoxicity elicited by cadmium-based quantum dots. Biomaterials. 2016;78:102-114.

54. Balmuri SR, Selvaraj U, Kumar VV, et al. Effect of surfactant in mitigating cadmium oxide nanoparticle toxicity: implications for mitigating cadmium toxicity in environment. Environ Res. 2017;152:141-149.

55. Pasquali F, Agrimonti C, Pagano L, et al. Nucleo-mitochondrial interaction of yeast in response to cadmium sulfide quantum dot exposure. J Hazard Mater. 2017;324(pt B):744-752.

56. Carvalho SMD, Mansur AAP, Mansur HS, Guedes MIMC, Lobato ZIP, Leite MF. In vitro and in vivo assessment of nanotoxicity of CdS quantum dot/aminopolysaccharide bionanoconjugates. Mater Sci Eng C Mater Biol Appl. 2017;71:412-424.

57. Tang H, Yang ST, Ke DM, et al. Blood clearance, distribution, transformation, excretion, and toxicity of near-infrared quantum dots $\mathrm{Ag} 2 \mathrm{Se}$ in mice. ACS Appl Mater Interfaces. 2016;8(28):17859-17869.

58. Vasudevan D, Gaddam RR, Trinchi A, Cole I. Core-shell quantum dots: properties and applications. J Alloys Comp. 2015;636:395-404.

59. Chan WC, Maxwell DJ, Gao X, Bailley RE, Han M, Nie S. Luminescent quantum dots for multiplexed biological detection and imaging. Curr Opin Biotechnol. 2002;13(1):40-46.

60. Goldman ER, Medintz IL, Mattoussi H. Luminescent quantum dots in immunoassays. Anal Bioanal Chem. 2006;384(3):560-563

61. Tiwari DK, Jin T, Behari L. Bio-distribution and toxicity assessment of intravenously injected anti-HER2 antibody conjugated CdSe/ZnS quantum dots in Wistar rats. Int J Nanomedicine. 2011;6:463-475. 
International Journal of Nanomedicine

Dovepress

\section{Publish your work in this journal}

The International Journal of Nanomedicine is an international, peerreviewed journal focusing on the application of nanotechnology in diagnostics, therapeutics, and drug delivery systems throughout the biomedical field. This journal is indexed on PubMed Central, MedLine, CAS, SciSearch ${ }^{\circledR}$, Current Contents ${ }^{\circledR} /$ Clinical Medicine,
Journal Citation Reports/Science Edition, EMBase, Scopus and the Elsevier Bibliographic databases. The manuscript management system is completely online and includes a very quick and fair peer-review system, which is all easy to use. Visit http://www.dovepress.com/ testimonials.php to read real quotes from published authors.

Submit your manuscript here: http://www.dovepress.com/international-journal-of-nanomedicine-journal 